\title{
Der Einfluss unterschiedlicher Vaterrassen in praxisüblichen Schweinekreuzungen auf deren Ansatzleistung, Schlachtkörper- zusammensetzung sowie Fleisch- und Fettbeschaffenheit
}

\author{
Herrn Professor Dr. Dr. h. c. Helmuth Pfeiffer zum 70. Geburtstag gewidmet
}

\begin{abstract}
Title of the paper: Effect of sire breeds in commercial pig crosses on growth, carcass composition, meat and fat quality

200 weaners from a large field trial in Niedersachsen to test different German and foreign sire breeds for their ability to improve the quality of market products were put at an individual feeding trial at the FAL-Nutrition Institute in Braunschweig. It was the aim of this feeding trial to compare the effects of different fat additions to the diet, sire breeds, sexes and slaughter weights upon growth and usual carcass traits but also upon several additional meat and fat quality traits. The trial was carried out in two blocks with 96 pigs each with the sire breeds Pietrain of the two MHS-genotypes PP (stress susceptible) and NN (stress resistant), Duroc and Hamsphire*Duroc (both NN) as preferrably used in Denmark. As control in both blocks the BHZP-standard boar $65(\mathrm{PI} * \mathrm{HA}(\mathrm{NN}))$ was used. Here only the sire breed effects will be reported. In daily gain and energy conversion only DU-progeny differed significantly from the control $(+33 g,-1.32 \mathrm{MJ} / \mathrm{kg})$. The tissue dissection showed that PI(PP)-, DU- and HA*DU-progeny had $0.8-1.0 \%$ higher bone and respectively lower residual components (lean, fat, tissue). In carcass measurements PI(PP) progeny were the leanest followed by PI(NN), and DU progeny had the smallest loin eye areas and carcass lean contents. This affected the carcass cuts such that PI(PP) progeny had a $1.2 \%$ (= $1.08 \mathrm{~kg}$ ) higher sum of primal cuts and DU progeny with $-0,8 \%(-400 \mathrm{~g})$ below the control were the poorest. In meat quality traits only the heterozygote stress susceptible PI(PP) progeny showed highly significant poorer values than all other crosses, which differed little from each other. This was confirmed by drip losses 2 and 4 days post mortem, which were $1.3-1.6 \%$ higher than the control whereas DU progeny were the best with $-0.6 \%$. Chemical analysis of a loin sample produced for both Danish sire breeds significantly higher intramuscular fat $(+0.36-0.38 \%)$ and for PI(PP) progeny with $-0.32 \%$ the lowest values, but the overall mean reached only $1.3 \%$ intramuscular fat. The protein and glycogen contents of loin revieled the expected large deviations of Hamsphire progeny due to the dominant $\mathrm{RN}^{-}$gene (-0.8-1 \% protein, $+10-14 \mu \mathrm{mol} / \mathrm{g}$ glycogen). All HA-free crossbreds did not differ much. The fatty acid composition of backfat and intramuscular fat showed much smaller differences between sire breeds than between fat supplements to the ration, but Danish sire breeds transmitted higher contents of polyunsaturated and Omega 3 fatty acids than the other sire breeds. Finally, for animals of the second trial also a sensoric test of loin samples was performed at the Federal Meat Research Institute. There subjective scores for tenderness and juiciness as well as grill losses showed significant sire breed differences. But contrary to expectation both Danish sire breeds produced inferior quality in all three traits to $\mathrm{PI} H \mathrm{HA}(\mathrm{NN})$ control sire groups.
\end{abstract}

Key Words: growth, carcass value, meat quality, German and Danish pig breeds

\section{Zusammenfassung}

Das Ziel dieses Fütterungsversuches am Tierernährungsinstitut der FAL in Braunschweig war es, die Einflüsse verschiedener Fettergänzungen zum Mastfutter, der Vaterrasse, des Geschlechts und des Mastendgewichts auf herkömmliche Kriterien der Fleischleistung und -beschaffenheit einerseits und zahlreiche weitergehende Fleisch- und Fettqualitätsindikatoren zu untersuchen. Der Versuch wurde in zwei Durchgängen zu je 96 Tieren mit den Vaterrassen zweimal Pietrain der MHS-Genotypen PP (stressanfällig) und NN (stressstabil) sowie den in Dänemark üblichen Duroc- und Hampshire*Duroc (beide NN)-Ebern durchgeführt. Als Kontrolle diente in bei- 
den Durchgängen der BHZP-Standardeber 65 (PI*HA(NN)). In dieser Publikation werden nur die auf die verschiedenen Vaterrassen zurückzuführenden Einflüsse vorgestellt. In der täglichen Lebendmassezunahme und im Energieaufwand unterschieden sich nur die DU-Nachkommen signifikant vom Standard (+33g bzw. - 1,32 MJME/kg). In der Gewebezerlegung zeigten PI(PP)-, DU- sowie HA*DU-Nachkommen um 1 \% bzw. 0,8 \% signifikant höhere Knochenanteile und entsprechend niedrigere „Restanteile“. Bei den Schlachtkörpermaßen waren PI(PP)-Nachkommen die fleischreichsten, gefolgt von PI(NN)- und DU-Nachkommen mit den niedrigsten Muskelflächen und Fleischanteilen in der Hälfte. Bei der Teilstückzerlegung hatten PI(PP)-Nachkommen einen um 1,2 \% (= $1.08 \mathrm{~kg}$ ) höheren und DU-Nachkommen einen um 0,8 \% ( = -400 g) geringeren Anteil wertvoller Teilstücke als die Kontrolle. In der Fleischbeschaffenheit lagen die mischerbig stressanfälligen PI(PP)-Nachkommen hoch signifikant unter allen anderen Gruppen, die sich untereinander kaum unterschieden. Bestätigt wurde dies durch die Dripverluste nach 2 bzw. 4 Tagen, die um 1,6 \% bzw. 1,3 \% höher lagen und bei DUNachkommen mit -0,6 \% am geringsten waren. Der intramuskuläre Fettgehalt im Kotelett ergab für beide dänischen Vaterrassen signifikant höhere (+0,36 bis 0,38 \%) und für PI(PP) niedrigere (-0,32 \%) Werte. Der Proteinund Glykogengehalt im Muskel zeigte die erwarteten deutlichen Abweichungen durch das bei Hamsphirenach-

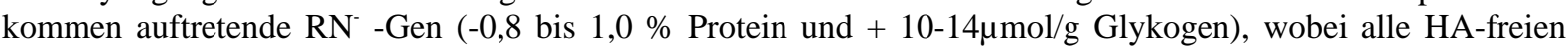
Tiere nicht signifikant verschieden waren. Die Fettsäurenzusammensetzung des Rückenspecks wie des intramuskulären Fetts zeigte zwischen Vaterrassen viel geringere Unterschiede als zwischen den Futterfettkomponenten, gesicherte Unterschiede zwischen den Kreuzungsgruppen wurden nur bei mehrfach ungesättigten und Omega 3-Fettsäuren gefunden. Schließlich wurde ebenfalls nur bei Tieren des zweiten Durchgangs ein sensorischer Test von Kotelettproben an der Bundesanstalt für Fleischforschung durchgeführt. Dabei zeigten nur die subjektiven Benotungen der Saftigkeit und Zartheit signifikante Rassenunterschiede. Entgegen der Erwartung zeigten beide dänischen Vaterrassen dabei ungünstigere Mittelwerte als die PI*HA-Kontrolltiere.

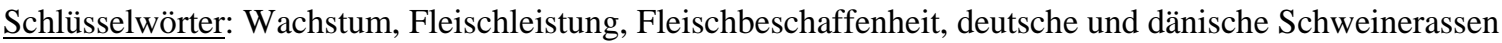

1.

\section{Einführung}

In den Jahren 1997-99 wurde in Niedersachsen mit Unterstützung des Landwirtschaftsministeriums und der beteiligten Wirtschaft ein umfangreicher Feldversuch (900 Würfe, 7000 Mastschweine) durchgeführt, um zu prüfen, wie die Qualität von Markenschweinen durch den Einsatz verschiedener Vaterrassen verbessert werden könnte (GLODEK, 1999; PAULUS et al. 2000; LAUBE et al., 2000). Aus diesem Feldversuch wurden 196 Absatzferkel fünf verschiedener Kreuzungskombinationen entnommen, um an ihnen in einem exakten Einzelfütterungsversuch individuelle Ansatz- und Qualitätsdaten bei verschiedenen Fettzusätzen zum Mastfutter zu gewinnen (KRATZ, 2003). Die Versuchsferkel wurden mit Sperma repräsentativ aus den beteiligten Vaterrassen ausgewählter Besamungseber mehrerer deutscher und einer dänischen KB-Station erzeugt und im Versuchsstall der FAL in Braunschweig in Einzelmast geprüft. Der Fütterungsversuch, die versuchsmäßige Ausschlachtung der Tiere wie die Gewinnung aller Wachstums-, Schlachtkörper- und Laboruntersuchungsdaten erfolgte im Institut für Tierernährung der FAL Braunschweig.

In dieser Publikation werden nur die Unterschiede zwischen den genetischen Gruppen, d.h. den verschiedenen Kreuzungskombinationen, vorgestellt, die futterbedingten Einflüsse werden an anderer Stelle publiziert. Systematische Störeffekte wie Geschlecht, Endgewicht und Saison wurden bei der Versuchsplanung und der statistischen Auswertung berücksichtigt.

Die genetische Zielsetzung bestand darin herauszuarbeiten, wie die Nachkommen von stressanfälligen und stressresistenten deutschen Pietrainebern [PI(PP), PI(NN)] und in Dänemark stark benutzten Duroc- (DU) und Hampshire*Duroc (HA*DU)-Ebern (alle stressresistent NN) im Vergleich zu denen von Standardebern PI*HA (ebenfalls NN) hinsichtlich möglichst vieler, über die normale Prüfungsroutine hinausgehender Leistungs- und Qualitätskriterien abschneiden. Dabei war davon auszugehen, dass die Hauptgene MHS(P) aus PI(PP)-Ebern und $\mathrm{RN}^{-}$aus HA-Reinzucht- wie Kreuzungs- 
ebern vor allem verschiedene Komplexe der Fleischbeschaffenheit und der hohe intramuskuläre Fettgehalt der DU-Eber möglicherweise die Genusseigenschaften des Fleisches ihrer Nachkommen beeinflussen würden.

\section{Material und Methoden}

Alle Versuchstiere stammten aus einem brandenburgischen Ferkelerzeugungsbetrieb, in welchem in zwei Durchgängen über je 20 Wochen wöchentlich 2 KB-Eber aus verschiedenen Vaterrassen auf je 10 BHZP-Hybridsauen eingesetzt wurden. Daraus konnten für die zwei Durchgänge dieses Einzelfütterungsversuches bei jeweils 8-wöchiger Anlieferung je 4 Väter aus drei Vaterrassen mit je 8 Nachkommen (je 1 Borg und 1 Sau aus 4 verschiedenen Würfen) ausgewählt werden. Der 1. Durchgang lief von Januar bis Juli 1998, der 2. von August 1998 bis Februar 1999, die Verteilung der Versuchstiere über Rassen, Väter, Geschlechter und Fütterungsgruppen geht aus Tabelle 1 hervor.

Tabelle 1

Verteilung der Versuchsferkel über Rassen, Geschlechter, Väter und Fütterungsgruppen (Distribution of pigs over sire breeds, sexes, sires and feed groups)

\begin{tabular}{|c|c|c|c|c|c|}
\hline \multirow[b]{2}{*}{ Durchgang } & \multirow[b]{2}{*}{ Vaterrasse } & \multirow[b]{2}{*}{ Väter } & \multicolumn{3}{|c|}{ Anzahl Nachkommen } \\
\hline & & & Börge & Sauen & insgesamt \\
\hline \multirow[t]{7}{*}{1} & $\mathrm{PI}(\mathrm{NN})$ & 4 & 16 & 16 & 32 \\
\hline & $\mathrm{PI}(\mathrm{PP})$ & 4 & 16 & 16 & 32 \\
\hline & PI*HA(NN) & 4 & 16 & 16 & 32 \\
\hline & Fütterungsgruppe & & & & \\
\hline & Standard & & 24 & 24 & 48 \\
\hline & + 2,5 \% Sojaöl & & 24 & 24 & 48 \\
\hline & Insgesamt & & 48 & 48 & 96 \\
\hline \multirow{9}{*}{2} & $\overline{\mathrm{DU}}$ & 4 & 16 & 16 & 32 \\
\hline & $\mathrm{HA} * \mathrm{DU}$ & 3 & 16 & 16 & 32 \\
\hline & PI*HA(NN) & 6 & 16 & 16 & 32 \\
\hline & Fütterungsgruppe & \multirow{5}{*}{ jeweils $+2,5 \%$} & & & \\
\hline & Rindertalg & & 12 & 12 & 24 \\
\hline & Olivenöl & & 12 & 12 & 24 \\
\hline & Sojaöl & & 12 & 12 & 24 \\
\hline & Leinöl & & 12 & 12 & 24 \\
\hline & Insgesamt & & 48 & 48 & 96 \\
\hline
\end{tabular}

Bis auf die Rasse HA*DU, in welcher irrtümlich derselbe Vater zweimal ausgewählt wurde, und 3 Ersatztiere von 2 zusätzlichen Vätern in der Kontrollgruppe PI*HA (NN) wurde der Versuchsplan eingehalten. Zwar konnte man mit nur 4 (3) zufällig ausgewählten Besamungsebern keine zuverlässigen Rassenvergleiche erwarten, jedoch war das Hauptziel dieses Exaktversuches, unter kontrollierten Bedingungen die an großem Feldmaterial mit praxisüblichen Merkmalen durchgeführten Vergleiche mithilfe zahlreicher individueller Wachstums- und Labormerkmale zu ergänzen.

$\mathrm{Zu}$ den kontrollierten Bedingungen gehörte die isoenergetische Futtervorlage nach Rationstabelle. Unterschiede im Fütterungsregime (DG1: zweiphasig mit 17,7 \% bzw. 15,5\% XP; DG2: einphasig mit 17,5 \% XP) führten zu einer um 0.6 MJ ME/Tag höheren Futteraufnahme im 2. Durchgang, womit die gefundene höhere Tageszunahme in etwa erklärt wird. Die Rassenvergleiche sind von dieser Differenz nicht betroffen, da in beiden Durchgängen mit Abweichungen von der gleichen Standardgruppe PI*HA gerechnet wurde. 
Die Wachstumsleistung wurde anhand folgender Merkmale bestimmt: Mastdauer (Tage) und Lebendmassezunahmen (g/Tag) im Abschnitt 30 - $120 \mathrm{~kg}$, aus Mastendgewicht lebend (LMZ), aus Zweihälftengewicht (NZ) und aus „Fleisch“ (Zweihälftengewicht - Knochengewicht) als FMZ. Schließlich wurde der ME-Aufwand je kg LMZ aus Futteraufnahme (kg/Tag) * Futterenergie (MJ ME/kg)/LMZ (g/Tag)/1000 berechnet.

Am Schlachtkörper wurden die in der Anstaltsprüfung üblichen Maße für Zweihälftengewicht (warm und kalt), Länge, Speckdicken (3 Rücken-, 1 Seitenspeckmaß), Muskel-, Fettfläche und Fleisch-Fett-Verhältnis daraus wie die Teilstückgewichte Schinken, Schulter, Kamm, Kotelett, Bauch gewonnen. Zusätzlich Schinken und Schulter schier, jeweils ohne Knochen, Speck und Sehnen. Berechnet wurden daraus die Schlachtausbeute [100 (warmes Zweihälftengewicht/Lebendgewicht)], Summe wertvoller Teilstücke (SWT) in kg (= Schinken (schier) + Schulter (schier) + Kotelett + Kamm) und ebenfalls in \% der linken Hälfte kalt. Außerdem wurden der Muskelfleischanteil im Schlachtkörper nach Bonner Formel (MFA/BF) und der Muskelfleischanteil im Bauch nach Gruber Formel (BFA/GF) berechnet.

Als Maße der Fleischbeschaffenheit wurden die pH-Werte im M. long. dorsi und semimembr. jeweils 40 Minuten und 24 h p.m. und die entsprechenden Leitfähigkeitswerte 45 Minuten und 24 h p.m. gemessen, außerdem die Fleischhelligkeit mit dem Opto-Star und der Drip (Tropfsaftverlust) jeweils, 2, 4, 6 und 8 Tage p.m..

Für Laboruntersuchungen wurden aus dem M.l.d. ab der 14. Rippe 3 Scheiben à $1 \mathrm{~cm}$ Dicke entnommen, je eine für Nährstoff-, Fettsäurenmuster- und Glykogenanalysen, vom Rückenspeck eine Probe durch alle Schichten an der 14. Rippe. Von 46 (48) Schweinen des 1. (2. ) Durchgangs wurden die rechten Hälften in Knochen und „Fleisch“ (= Muskel + Fett + Schwarte + Bindegewebe) manuell zerlegt, außerdem an acht $30 \mathrm{~kg}$-Tieren, der sog. Nullgruppe, um den Zuwachs berechnen zu können. In die Nährstoffanalysen gingen Trockensubstanz (T), Rohprotein (XP), Rohfett (XL) sowie Asche (XA) ein. Das Fettsäurenmuster wurde im Kotelett und Rückenspeck untersucht, der Glykogengehalt in den M.l.d.-Proben und die Oxidationsstabilität mit dem Rancimat-Test in den Rückenspeckproben. Schließlich wurde an 67 Tieren des Durchgangs 2 von einem Team der Bundesanstalt für Fleischforschung (BAFF) ein sensorischer Test nach Fischer (GLODEK, 1999; KRATZ, 2003) durchgeführt. Die Analysen erfolgten nach VDLUFA-Methoden, weitere Details, auch zum Sensoriktest der BAFF, wurden von KRATZ (2003) veröffentlicht.

Die statistische Auswertung der Daten erfolgte mit dem GLM-Paket von SAS/STAT, Version 6.12. In das Modell gingen die Hauptfaktoren: Vaterrasse, Futtergruppe, Geschlecht und im Durchgang 1 noch die Gewichtsklasse ein, sowie alle signifikanten Zweifach-Interaktionen. Wenn im folgenden vereinfachend von „Rassenunterschieden“ gesprochen wird, so sind die Unterschiede zwischen den Kreuzungsendprodukten der verschiedenen Vaterrassen gemeint. Bei Wachstumsmerkmalen wurden das Mastendgewicht, bei Schlachtkörpermerkmalen das Zweihälftengewicht als Kovariablen in das Modell einbezogen, im 1. Durchgang mit zwei vorgegebenen Endgewichten jeweils die Differenz zu deren Mitteln. Von KRATZ (2003) wurden beide Durchgänge getrennt ausgewertet, wobei für im F-Test signifikante Rassenunterschiede ein Scheffé-Test zwischen den jeweils drei Rassenmitteln durchgeführt wurde. Bei nicht normal verteilten Merkmalen wurde der Median statt des Mittels angegeben, und die Rassenunterschiede wurden mit dem $\chi^{2}$-Test geprüft. 
Hier geht es um den Vergleich der vier „Versuchsrassen“ untereinander, was nur anhand ihrer Abweichungen von der jeweiligen Durchgangskontrollgruppe (PI*HA) möglich ist. In den nachfolgenden Ergebnistabellen sind diese Abweichungen mit approximierten Standardfehlern angegeben. Zur Abschätzung der Bedeutung dieser Abweichungen sind die jeweiligen Durchgangsrohmittel sowie die Differenz der Standardgruppenmittel (VG1 - VG2) angegeben sowie die Signifikanz der Rassenunterschiede innerhalb Durchgängen im F-Test in der üblichen *-Notation.

\section{Ergebnisse}

\section{Zuwachsleistungen und Energie-Aufwand}

Die Rassenvergleiche zu diesem Leistungskomplex sind in Tabelle 2 zusammengestellt.

Hinsichtlich Mastdauer, Lebendmassezunahme und ME-Aufwand fanden sich signifikante Unterschiede nur zwischen den im zweiten Durchgang geprüften Rassen. Nachkommen von stressanfälligen und -resistenten PI-Ebern zeigten keine signifikant verschiedenen Mastleistungen in diesem zahlenmäßig begrenzten Datenmaterial, jedoch lagen die absoluten Differenzen durchaus im praktisch relevanten Bereich und zwar zugunsten der stressanfälligen PI-Eber. Dagegen brachten DU-Nachkommen hoch signifikant bessere Mittelwerte in allen drei Wachstumskriterien, und (HA*DU)Nachkommen lagen im nicht mehr signifikanten Mittelfeld.

Betrachtet man die im Teilmaterial ermittelten Zerlegedaten im unteren Teil von Tabelle 2, so fallen die hoch signifikanten Unterschiede im Knochen- und „Restanteil“ in beiden Durch-gängen auf. Sie beruhen darauf, dass Nachkommen von PI(PP)- und DU-Ebern um 1 \% höhere Knochen- und entsprechend niedrigere Anteile der aufsummierten restlichen Gewebe aufweisen. Zwar finden sich in der Nettozunahme für beide Vaterrassen noch signifikante Überlegenheiten, aber bezogen auf die knochenfreien Gewebeanteile verschwinden diese weitgehend. Die höheren Brutto- wie Nettozunahmen beruhen also im wesentlichen auf einem höheren Knochenzuwachs bei PI(PP)-, DU- und auch (HA*DU)-Nachkommen. Der höhere Anteil an Restgeweben bei PI(NN)- und (PI*HA)-Nachkommen wird allerdings anhand der Schlachtkörperzusammensetzung aus echtem Muskelfleisch und Fett weiter zu analysieren sein.

\section{Schlachtkörpermaße und Teilstückanteile}

Die Rassenvergleiche für die wichtigsten Merkmale in diesem Leistungsbereich sind in Tabelle 3 wiedergegeben. Signifikante Rassenunterschiede finden sich bei den Schlachtkörpermaßen Schlachtkörperlänge, Rückenmuskel- und Fettfläche, FleischFett-Verhältnis und dem geschätzten Fleischanteil in der Hälfte und im Bauch.

PI(NN)-Eber erzeugten die kürzesten und DU-Eber, gefolgt von HA*DU-Ebern, die längsten Nachkommen. Entsprechend hatten erstere die größten und letztere die kleinsten Rückenmuskelflächen, wobei DU-Nachkommen mit $4 \mathrm{~cm}^{2}$ unter dem Vergleichsstandard deutlich abfielen. Da sie aber die kleinsten Auflage-Fettflächen besaßen, unterschieden sie sich im Fleisch-Fett-Verhältnis nicht vom Standard, während darin die PI(PP)-Nachkommen hoch signifikant besser waren als alle anderen Rassen. Im geschätzten Fleischanteil der Hälften führten die PI(PP)-Nachkommen und lagen über 2 \% über den am Ende rangierenden DU-Nachkommen. Im geschätzten Fleischanteil des Bauches führten ebenfalls die PI(PP)-Nachkommen hoch signifikant, aber darin lagen die dänischen Kreuzungen leicht über und die PI(NN)-Nachkommen als 
Tabelle 2

Zuwachsleistungen an Lebend-, Schlacht- und „Fleisch“-Gewicht sowie ME-Aufwand je kg Zuwachs (Abweichungen von den jeweiligen Vergleichsstandards). (Growth performance in live-, carcass- and „meat“ weight and ME consumption per kg growth (in MJ) (Deviations from standard PI*HA(NN))

\begin{tabular}{|c|c|c|c|c|c|c|c|c|c|}
\hline \multirow[b]{2}{*}{ Merkmal } & \multicolumn{2}{|c|}{ F-Test } & \multicolumn{4}{|c|}{ Abweichung vom VG-Standard } & \multirow{2}{*}{$\begin{array}{c}\text { Differenz } \\
\text { VG1-VG2 }\end{array}$} & \multicolumn{2}{|c|}{ Rohmittel (s) } \\
\hline & $\begin{array}{c}\text { Zwischen } \\
\text { D1 }\end{array}$ & $\begin{array}{c}\text { Rassen } \\
\text { D2 }\end{array}$ & $\mathrm{PI}(\mathrm{NN})$ & $\mathrm{PI}(\mathrm{PP})$ & DU & HA*DU & & D1 & D2 \\
\hline $\bar{N}$ & & & 32 & 32 & 32 & 32 & Je 31 & 95 & 96 \\
\hline Mastdauer (Tage) & - & $*$ & $+2,3 \pm 2,0$ & $+0,8 \pm 1,8$ & $-3,8 \pm 0,9$ & $-1,2 \pm 1,3$ & $-0,3$ & $105(11)$ & $104(6)$ \\
\hline Tägl. Zunahme (g) & - & * & $-12 \pm 12$ & $+12 \pm 10$ & $+33 \pm 8$ & $+7 \pm 10$ & $-42 * *$ & $814(57)$ & $870(48)$ \\
\hline ME-Aufwand (MJ/kg) & - & $*$ & $+0,17 \pm 0,59$ & $-0,74 \pm 0,53$ & $-1,32 \pm 0,35$ & $-0,48 \pm 0,26$ & $+0,73$ & $36,8(3,1)$ & $35,0(2,1)$ \\
\hline $\mathrm{N}$ & & & 15 & 16 & 16 & 16 & & 46 & 48 \\
\hline \% Knochen von ZHG & & $* *$ & $-0,1 \pm 0,3$ & $+1,0 \pm 0,2$ & $+1,0 \pm 0,2$ & $+0,8 \pm 0,3$ & $-0,6^{\circ}$ & $10,2(1,0)$ & $11,1(0,9)$ \\
\hline$\%$ „Fleisch“ & $* *$ & $* *$ & $+0,1 \pm 0,3$ & $-0,9 \pm 0,2$ & $-1,0 \pm 0,2$ & $-0,8 \pm 0,3$ & $+0,6^{\circ}$ & $88,9(1,0)$ & $89,8(1,0)$ \\
\hline Netto Zunahme g (v. ZHG) & $* *$ & - & $+6 \pm 15$ & $+27 \pm 12$ & $+19 \pm 9$ & $+12 \pm 9$ & $-34^{*}$ & $663(5,1)$ & $697(31)$ \\
\hline \% von tägl. Zunahme & - & & $+1,2$ & $+1,8$ & $-2,0$ & $-1,3$ & $+1,0$ & 82,8 & 80,7 \\
\hline „Fleisch“-Zunahme g (v. ZHG) & & - & $+8 \pm 14$ & $+17 \pm 11$ & $+8 \pm 5$ & $+5 \pm 6$ & $-27^{\circ}$ & $604(47)$ & $626(29)$ \\
\hline \% v. tägl. Zunahme & - & & $+1,3$ & $+0,7$ & $-1,1$ & $-0,7$ & $+0,5$ & 75,4 & 72,5 \\
\hline
\end{tabular}

\% v. tägl. Zunahme

$-$

D1 , D2 = Durchgänge 1, 2; VG1, VG2 = Vergleichsstandard (PI*HA(NN)) 1, 2

Die Standardfehler der Abweichungen sind Näherungswerte

„Fleisch“= Hälftenkaltgewicht - Knochengewicht 
Tabelle 3

Schlachtkörpermaße und Teilstückanteile (Abweichungen von den jeweiligen Vergleichsstandards) (Carcass measurements and cuts (Deviations from standard PI*HA(NN))

\begin{tabular}{|c|c|c|c|c|c|c|c|c|c|}
\hline \multirow[b]{2}{*}{ Merkmal } & \multicolumn{2}{|c|}{ F-Test } & \multicolumn{4}{|c|}{ Abweichung vom VG-Standard } & \multirow{2}{*}{$\begin{array}{c}\text { Differenz } \\
\text { VG1-VG2 }\end{array}$} & \multicolumn{2}{|c|}{ Rohmittel (s) } \\
\hline & $\begin{array}{c}\text { Zwischen } \\
\text { D1 }\end{array}$ & $\begin{array}{c}\text { Rassen } \\
\text { D2 } \\
\end{array}$ & $\mathrm{PI}(\mathrm{NN})$ & $\mathrm{PI}(\mathrm{PP})$ & DU & HA*DU & & D1 & D2 \\
\hline $\mathrm{N}$ & & & 32 & 32 & 32 & 32 & je 31 & 95 & 96 \\
\hline Schlachtkörperlänge (cm) & $*$ & $* *$ & $-1,0 \pm 0,34$ & $+0,0 \pm 0,35$ & $+1,9 \pm 0,42$ & $+1,6 \pm 0,30$ & $+0,3$ & $99,7(1,8)$ & $100,9(2,3)$ \\
\hline Rückenspeckdicke (cm) & - & - & $+0,06 \pm 0,06$ & $-0,09 \pm 0,06$ & $+0,03 \pm 0,05$ & $-0,08 \pm 0,05$ & $+0,1$ & $2,4(0,3)$ & $2,3(0,3)$ \\
\hline Rückenmuskelfläche ( $\left.\mathrm{cm}^{2}\right)$ & - & $*$ & $+2,4 \pm 1,2$ & $+2,3 \pm 0,9$ & $-4,0 \pm 1,1$ & $-1,8 \pm 0,9$ & $-2,6$ & $50,2(5,8)$ & $49,3(5,9)$ \\
\hline Fett-Fläche $\left(\mathrm{cm}^{2}\right)$ & $* *$ & $*$ & $+1,0 \pm 0,6$ & $-1,9 \pm 0,7$ & $-2,3 \pm 0,5$ & $-1,3 \pm 0,7$ & $-0,3$ & $19,8(4,2)$ & $20,2(3,4)$ \\
\hline Fleisch:Fett Verhältnis & $* *$ & - & $+0,001 \pm 0,0017$ & $-0,059 \pm 0,016$ & $-0,009 \pm 0,017$ & $-0,012 \pm 0,016$ & $-0,001$ & $0,400(0,11)$ & $0,415(0,09)$ \\
\hline MFA (BF) \% & $*$ & - & $+0,5 \pm 0,4$ & $+1,4 \pm 0,4$ & $-0,7 \pm 0,4$ & $-0,1 \pm 0,3$ & $-0,7$ & $58,7(2,0)$ & $58,6(2,1)$ \\
\hline BFA (GF) \% & $*$ & - & $-0,2 \pm 0,5$ & $+1,8 \pm 0,7$ & $+0,4 \pm 0,4$ & $+0,7 \pm 0,4$ & $-0,3$ & $57,0(2,9)$ & $57,2(2,6)$ \\
\hline Schinken \% & $*$ & $*$ & $+0,2 \pm 0,19$ & $+0,6 \pm 0,19$ & $+0,1 \pm 0,16$ & $-0,4 \pm 0,14$ & $-0,6^{* *}$ & $30,0(1,0)$ & $30,2(0,9)$ \\
\hline Schinken schier \% & - & - & $+0,1 \pm 0,21$ & $+0,8 \pm 0,23$ & $-0,3 \pm 0,19$ & $-0,1 \pm 0,16$ & $-1,0 * *$ & $16,9(1,1)$ & $17,5(1,0)$ \\
\hline Kotelett \% & - & - & $+0,1 \pm 0,14$ & $+0,2 \pm 0,12$ & $-0,4 \pm 0,18$ & $-0,1 \pm 0,16$ & $-0,6^{* *}$ & $12,3(0,7)$ & $12,6(0,9)$ \\
\hline Kamm \% & - & - & $-0,1 \pm 0,09$ & $\pm 0 \pm 0,07$ & $+0,2 \pm 0,12$ & $+0,3 \pm 0,21$ & $-0,6 * *$ & $7,4(0,5)$ & $8,2(0,9)$ \\
\hline Schulter \% & - & $*$ & $+0,2 \pm 0,18$ & $+0,5 \pm 0,16$ & $\pm 0 \pm 0,14$ & $+0,4 \pm 0,11$ & $-1,0 * *$ & $12,6(0,8)$ & $13,6(0,7)$ \\
\hline Schulter schier \% & - & $\circ$ & $+0,2 \pm 0,09$ & $+0,3 \pm 0,12$ & $-0,1 \pm 0,11$ & $+0,2 \pm 0,07$ & $-0,3^{*}$ & $7,9(0,6)$ & $8,5(0,5)$ \\
\hline Bauch \% & - & $*$ & $\pm 0 \pm 0,12$ & $-0,2 \pm 0,14$ & $+0,5 \pm 0,16$ & $+0,2 \pm 0,11$ & $+0,9 * *$ & $14,8(1,0)$ & $13,6(0,8)$ \\
\hline Anteil wertv. Tst. \% & - & $*$ & $+0,2 \pm 0,35$ & $+1,2 \pm 0,39$ & $-0,8 \pm 0,25$ & $+0,2 \pm 0,34$ & $-2,7 * *$ & $447(1,9)$ & $46,7(1,6)$ \\
\hline Anteil wertv. Tst. kg & - & $*$ & $+0,41 \pm 0,25$ & $+1,08 \pm 0,25$ & $-0,40 \pm 0,14$ & $\pm 0 \pm 0,18$ & $-2,31 * *$ & $20,0(0,8)$ & $21,6(0,9)$ \\
\hline
\end{tabular}


schlechteste leicht unter dem Standard.

Die Ergebnisse der Teilstückzerlegung sind möglicherweise durch die hochsignifikant besseren Standardtiere des 2. Durchgangs beeinflusst, was besonders in der Summe der wertvollen Teilstücke mit $+2,7 \%$ (entspricht 2,31 kg) sichtbar wird. Im wesentlichen passen die gefundenen Abweichungen aber zu den Schlachtkörpermaßen. Im prozentualen Anteil wertvoller Teilstücke lagen die PI(PP)-Nachkommen 1,2 \% (bzw. $1,1 \mathrm{~kg}$ ) über der Standardgruppe und die DU-Nachkommen 0,8 \% (bzw. 0,4 kg) unter dieser, beides hoch signifikant. PI(NN)-Nachkommen waren in beiden Merkmalen überdurchschnittlich, aber deutlich schlechter als die ihrer stressanfälligen Geschwister. Bei den einzelnen Teilstücken gab es vor allem im Schinken-, Schulter- und Bauchanteil signifikante Rassenunterschiede, während im Kotelett lediglich die DUNachkommen um 0,4 kg unter dem Standard lagen.

Den höchsten Schinkenanteil hatten PI(PP)-Nachkommen, den niedrigsten HA*DUNachkommen mit $1 \%$ weniger. Beim schieren Schinken waren erstere noch besser, aber am Ende rangierten DU-Nachkommen mit 1,1 \% weniger. PI(PP)- und (HA*DU)-Nachkommen hatten die höchsten Schulteranteile, bei schieren Schultern halbierten sich die Überlegenheiten. Im Bauchanteil waren nur DU-Nachkommen mit $+0,5 \%$ signifikant abweichend.

Insgesamt hatten die stressanfälligen PI(PP)-Nachkommen die fleischreichsten Schlachtkörper und die von DU-Ebern lagen insbesondere in der Rückenmuskelfläche, im geschätzten Fleischanteil der Hälfte, im Kotelettanteil und im Anteil wertvoller Teilstücke hoch signifikant mit $-2 \%$ (entsprechend $-1,5 \mathrm{~kg}$ ) am Ende der Skala. Nachkommen stressresistenter PI(NN)-Eber waren am kürzesten und lagen trotz höchster Rückenmuskelfläche im geschätzten Fleischanteil der Hälfte um 0,9 \% unter denen von PI(PP)-Ebern. In den Teilstückanteilen lagen sie meist an zweiter Stelle hinter letzteren, in der Summe wertvoller Teilstücke allerdings um $1 \%$ oder 0,67 kg pro Mastschwein.

\section{Fleischbeschaffenheit}

Die wichtigsten Hilfsmerkmale der Fleischbeschaffenheit sind in Tabelle 4 als Rassenabweichungen vom Vergleichsstandard dargestellt.

Auch hier gab es z.T. signifikante Unterschiede zwischen den Vergleichsstandards im 1. und 2. Durchgang, aber die erwarteten Rassenunterschiede kamen sehr deutlich zum Ausdruck. In den PSE-relevanten Kriterien PH 40', LF45' und LF24h sowie im Tropfsaftverlust wichen die PI(PP)-Nachkommen, die alle den MHS-Status NP (mischerbig stressanfällig) hatten, hoch signifikant und deutlich von allen anderen reinerbig stressstabilen Rassegruppen ab, die ihrerseits untereinander weit weniger differierten.

Keine signifikanten Rassenunterschiede fanden sich im PH24h, und bei den Helligkeitsmessungen mit dem OPTOSTAR war die Messgenauigkeit unzureichend, wie die großen Durchgangsunterschiede zeigen.

Beim intramuskulären Fettgehalt zeigte die Standardgruppe im 1. Durchgang den höchsten und im zweiten den niedrigsten Wert, dennoch bilden die Abweichungen den bekannten Befund, dass die fleischreichsten Schweine den geringsten IMF besitzen, korrekt ab. Hier lagen die PI-Nachkommen (besonders die NP-Tiere) mehr als eine Standardabweichung unter den beiden dänischen Herkünften, die ihrerseits etwa gleichauf liegend nur ein Mittel von 1,74 \% erreichten.

Hinsichtlich des dominanten Hampshirefaktors wurde ganz deutlich, dass alle hampshirefreien Herkünfte hochsignifikant niedrigere Glykogen- und höhere Proteingehalte 
Tabelle 4

Hilfsmerkmale der Fleischbeschaffenheit (Abweichungen von den jeweiligen Vergleichsstandards) (Meat quailty traits (Deviations from standard PI*HA(NN))

\begin{tabular}{|c|c|c|c|c|c|c|c|c|c|}
\hline \multirow[b]{2}{*}{ Merkmal } & \multicolumn{2}{|c|}{ F-Test } & \multicolumn{4}{|c|}{ Abweichung vom VG-Standard } & \multirow{2}{*}{$\begin{array}{c}\text { Differenz } \\
\text { VG1-VG2 }\end{array}$} & \multicolumn{2}{|c|}{ Rohmittel (s) } \\
\hline & $\begin{array}{c}\text { Zwischen } \\
\text { D1 }\end{array}$ & $\begin{array}{c}\text { Rassen } \\
\text { D2 }\end{array}$ & $\mathrm{PI}(\mathrm{NN})$ & $\mathrm{PI}(\mathrm{PP})$ & DU & $\mathrm{HA} * \mathrm{DU}$ & & D1 & D2 \\
\hline $\mathrm{N}$ & & & 32 & 32 & 32 & 32 & je 31 & 95 & 96 \\
\hline PH40' m.l.d. & $* * *$ & - & $-0,06 \pm 0,04$ & $-0,46 \pm 0,06$ & $+0,11 \pm 0,06$ & $-0,01 \pm 0,04$ & $+0,26 * *$ & $6,21(0,35)$ & $6,16(0,25)$ \\
\hline PH40' m.s. & $* * *$ & $* *$ & $-0,08 \pm 0,04$ & $-0,55 \pm 0,06$ & $+0,4 \pm 0,05$ & $-0,18 \pm 0,05$ & $+0,30 * *$ & $6,31(0,37)$ & $6,17(0,31)$ \\
\hline LF45' m.l.d. & $* *$ & $* *$ & $-0,1 \pm 0,05$ & $+0,8 \pm 0,41$ & $\pm 0 \pm 0,07$ & $+0,1 \pm 0,13$ & $-0,1$ & $3,9(1,4)$ & $3,8(0,5)$ \\
\hline LF45' m.s. & $* * *$ & $*$ & $\pm 0 \pm 0,09$ & $+2,0 \pm 0,72$ & $-0,1 \pm 0,14$ & $+0,4 \pm 0,32$ & $-0,8^{* *}$ & $4,9(2,6)$ & $5,2(1,2)$ \\
\hline PH24 ${ }^{\mathrm{h}}$ m.l.d. & - & - & $-0,2 \pm 0,02$ & $-0,06 \pm 0,03$ & $-0,4 \pm 0,03$ & $-0,01 \pm 0,03$ & $-0,06$ & $5,53(0,17)$ & $5,61(0,17)$ \\
\hline $\mathrm{PH} 24^{\mathrm{h}} \mathrm{m} . \mathrm{s}$ & - & - & $-0,01 \pm 0,02$ & $+0,01 \pm 0,03$ & $-0,02 \pm 0,03$ & $\pm 0 \pm 0,03$ & $-0,10^{*}$ & $5,52(0,17)$ & $5,61(0,17)$ \\
\hline LF24 ${ }^{\mathrm{h}}$ m.l.d. & $* * *$ & $* * *$ & $-0,1 \pm 0,27$ & $+2,5 \pm 0,37$ & $+0,9 \pm 0,39$ & $+0,2 \pm 0,39$ & $-0,8^{* *}$ & $4,2(2,0)$ & $4,4(1,9)$ \\
\hline LF24 ${ }^{\mathrm{h}}$ m.s. & $* *$ & $* *$ & $+1,0 \pm 0,57$ & $+2,8 \pm 0,58$ & $+1,2 \pm 0,46$ & $\pm 0 \pm 0,46$ & $-3,0 * *$ & $8,3(3,5)$ & $10,5(2,7)$ \\
\hline OPTO $0 \mathrm{~d}$ & - & - & $-1,5 \pm 1,1$ & $-5,0 \pm 1,9$ & $+1,0 \pm 1,2$ & $+2,6 \pm 1,0$ & $+5,5^{* *}$ & $64,8(8,0)$ & $62,7(6,4)$ \\
\hline OPTO 4 d & - & $\circ$ & $+1,6 \pm 1,0$ & $-3,7 \pm 2,1$ & $+4,0 \pm 1,2$ & $+2,8 \pm 1,0$ & $+6,7 * *$ & $64,5(8,8)$ & $60,6(7,3)$ \\
\hline OPTO $8 \mathrm{~d}$ & - & - & $+0,6 \pm 1,1$ & $-2,7 \pm 1,7$ & $+1,8 \pm 1,0$ & $+1,7 \pm 1,1$ & $+3,3$ & $68,1(7,9)$ & $66,8(7,3)$ \\
\hline DRIP $2 \mathrm{~d} \%$ & $* *$ & - & $+0,6 \pm 0,42$ & $+1,6 \pm 0,28$ & $-0,6 \pm 0,37$ & $+0,04 \pm 0,34$ & $-0,8$ & $4,1(7,9)$ & $4,1(2,0)$ \\
\hline DRIP 4 d \% & $*$ & - & $+0,4 \pm 0,46$ & $+1,3 \pm 0,27$ & $-0,6 \pm 0,37$ & $+0,2 \pm 0,33$ & $-0,7$ & $5,5(2,1)$ & $5,7(2,0)$ \\
\hline DRIP $8 \mathrm{~d} \%$ & - & - & $+0,1 \pm 0,46$ & $+0,9 \pm 0,27$ & $-0,2 \pm 0,42$ & $0,3 \pm 0,33$ & $-0,3$ & $7,1(2,1)$ & $7,3(1,2)$ \\
\hline Gehalt im m.l.d. & & & & & & & & & \\
\hline Wasser $^{1)}(\%)$ & $* * *$ & $* * *$ & $-0,6 \pm 0,09$ & $-0,5 \pm 0,07$ & $-1,0$ & $-0,5$ & $-0,5^{*}$ & $74,8(0,6)$ & $75,1(0,6)$ \\
\hline Fett $^{1)}(\%)$ & $*$ & $* * *$ & $-0,18$ & $-0,32$ & $+0,38$ & $+0,36$ & $+0,27 *$ & $1,22(0,41)$ & $1,39(0,48)$ \\
\hline Protein ${ }^{1)}(\%)$ & $* * *$ & $*$ & $+0,8$ & $+1,0$ & $+1,0$ & $+0,2$ & $+0,5^{*}$ & $23,5(0,9)$ & $23,1(1,0)$ \\
\hline Glykogen $(\mu \mathrm{mol} / \mathrm{g})$ & $* * *$ & $* * *$ & $-13,7 \pm 0,25$ & $-14,7 \pm 0,11$ & $-10,9 \pm, 021$ & $-0,7 \pm 2,79$ & $+3,0$ & $6,1(11,8)$ & $8,6(13,8)$ \\
\hline
\end{tabular}


Tabelle 5

Die Fettsäurenmuster und die Oxidationsstabilität des Rückspecks (Abweichungen von den jeweiligen Vergleichsstandards) (Fatty acid composition and oxidation stability of backfat (Deviations from standard PI*HA(NN))

\begin{tabular}{|c|c|c|c|c|c|c|c|c|c|c|}
\hline \multirow[b]{2}{*}{ Merkmal } & & \multicolumn{2}{|c|}{ F-Test } & \multicolumn{4}{|c|}{ Abweichung vom VG-Standard } & \multirow{2}{*}{$\begin{array}{c}\text { Differenz } \\
\text { VG1-VG2 }\end{array}$} & \multicolumn{2}{|c|}{ Rohmittel (s) } \\
\hline & & $\begin{array}{l}\text { Zwischen } \\
\text { D1 }\end{array}$ & $\begin{array}{r}\text { Rassen } \\
\text { D2 } \\
\end{array}$ & $\mathrm{PI}(\mathrm{NN})$ & $\mathrm{PI}(\mathrm{PP})$ & DU & HA*DU & & D1 & D2 \\
\hline $\mathrm{N}$ & & & & 32 & 32 & $32(31)$ & 32 & je 32 & $96(95)$ & 96 \\
\hline SAFA & $\%$ & - & & $+0,6 \pm 0,41$ & $+0,1 \pm 0,41$ & $+0,2 \pm 0,49$ & $-0,3 \pm 0,37$ & $+4,4^{* *}$ & $44,1(2,2)$ & $39,4(2,6)$ \\
\hline MUFA & $\%$ & $\circ$ & - & $\pm 0 \pm 0,60$ & $-0,8 \pm 0,72$ & $-1,4 \pm 0,95$ & $-1,5 \pm 1,08$ & $-2,4^{\circ}$ & $42,6(3,8)$ & $44,2(6,1)$ \\
\hline PUFA & $\%$ & $* *$ & - & $-0,6 \pm 0,87$ & $+0,7 \pm 1.00$ & $+1,1 \pm 1,15$ & $+1,7 \pm 1,2$ & $-2,1$ & $13,3(5,3)$ & $16,3(6,5)$ \\
\hline Omega3 & $\%$ & $* *$ & $* *$ & $-0,1 \pm 0,09$ & $\pm 0 \pm 0,11$ & $+0,3 \pm 0,69$ & $+0,4 \pm 0,71$ & $-1,8 * *$ & $1,1(0,5)$ & $3,4(3,8)$ \\
\hline C16.0 & $\%$ & - & $* *$ & $+0,2 \pm 0,25$ & $+0,1 \pm 0,28$ & $-0,5 \pm 0,28$ & $-0,7 \pm 0,23$ & $+2,5 * *$ & $25,9(1,5)$ & $23,0(1,4)$ \\
\hline C16.1 & $\%$ & - & $*$ & $-0,1 \pm 0,07$ & $-0,1 \pm 0,09$ & $-0,3 \pm 0,07$ & $-0,3 \pm 0,09$ & \pm 0 & $2,3(0,5)$ & $2,2(0,4)$ \\
\hline C18.0 & $\%$ & - & $* * *$ & $+0,4 \pm 0,25$ & $+0,1 \pm 0,18$ & $+0,7 \pm 0,23$ & $+0,5 \pm 0,25$ & $+1,6^{* *}$ & $15,0(1,2)$ & $13,6(1,4)$ \\
\hline C18.1 & $\%$ & $*$ & - & $+0,1 \pm 0,55$ & $-0,8 \pm 0,62$ & $-1,1 \pm 0,90$ & $-1,1 \pm 0,99$ & $-2,3^{* *}$ & $39,4(3,3)$, & $41,2(5,6)$ \\
\hline C18.2 & $\%$ & $* *$ & - & $-0,4 \pm 0,74$ & $+0,7 \pm 0,85$ & $+0,7 \pm 0,72$ & $+1,3 \pm 0,80$ & $-0,1$ & $11,4(4,5)$ & $12,1(4,3)$ \\
\hline C18.3 & $\%$ & $*$ & $*$ & $-0,1 \pm 0,09$ & $+0,1 \pm 0,11$ & $+0,4 \pm 0,67$ & $+0,4 \pm 0,69$ & $-1,8 * *$ & $1,1(0,5)$ & $3,2(3,7)$ \\
\hline Induktio & nszeit h & - & $\begin{array}{l}* * \\
-\end{array}$ & $+0,3 \pm 0,53$ & $-0,3 \pm 0,37$ & $-0,3 \pm 0,27$ & $-0,1 \pm 0,34$ & $+1,7^{*}$ & $5,0(2,7)$ & $3,2(1,6)$ \\
\hline
\end{tabular}

SAFA = gesättigte-, MUFA = einfach-, PUFA = mehrfach ungesättigte Fettsäuren

Induktionszeit = Oxidationsstabilität bestimmt im Rancimat-Test (Methrom AG, Herisau) 
im Muskel aufwiesen, wobei sie sich untereinander genau wie die hampshiretragenden Kreuzungen wenig unterschieden. Erwähnt werden sollte, dass an diesem Material der End-PH-Wert diesbezüglich nicht aussagefähig war.

\section{Fettsäurenmuster im Rückenspeck}

Wesentliche Parameter des Fettsäurenmusters im Rückenspeck sind in Tabelle 5 wiedergegeben.

Zwar zeigte die Varianzanalyse im F-Test signifikante Rassenunterschiede, vor allem für PUFA, Omega3 und einige ungesättigte Fettsäuren, aber gleichzeitig wurden im zweiten Durchgang signifikante Interaktionseffekte zwischen Vaterrasse * Fütterungsgruppe für SAFA- MUFA-, PUFA-, C16.0- und C18.1-Fettsäuren gefunden und gar hoch signifikante für die Omega3- und C18.3-Fraktionen. Die Interpretation einzelner Rassenabweichungen wird daher durch sehr hohe Schätzfehler erschwert. Dennoch ist deutlich zu erkennen, dass DU- und HA*DU-Nachkommen höhere PUFA- und Omega3-Gehalte, vor allem auf Kosten der MUFA-Gehalte, besaßen als die PI- und PI*HA-Nachkommen. Von den einzelnen Fettsäuren trugen besonders die C16.0, C16.1 und die C18.1, C18.2 und z.T. auch die C18.3-Fraktionen zu diesem Bild bei.

Außerordentlich große Unterschiede zwischen den Standardrassen beider Durchgänge im Rancimat-Test für die Oxidationsstabilität des Rückenspecks dürften zwar zu erheblichen Teilen auf die gezeigten Unterschiede im Gehalt ungesättigter Fettsäuren zurückzuführen sein, jedoch scheint die Messgenauigkeit dieser Methode ebenfalls dazu beigetragen zu haben, dass die gefundenen Differenzen statistisch nicht abzusichern waren.

\section{Fettsäurenmuster des intramuskulären Fetts}

Nur im zweiten Durchgang wurde an 84 Tieren eine Probe aus dem Rückenmuskel auf den intramuskularen Fettgehalt und dessen Fettsäurenmuster untersucht, die Ergebnisse zeigt Tabelle 6.

\section{Tabelle 6}

Fettsäurenmuster des intramuskulären Fettgehalts (nur 2. Durchgang) (Abweichung vom VG-Standard) (Fatty acid composition of intramuscular fat in M. long. dorsi (only $2^{\text {nd }}$ trial) (Deviation from standard PI*HA(NN))

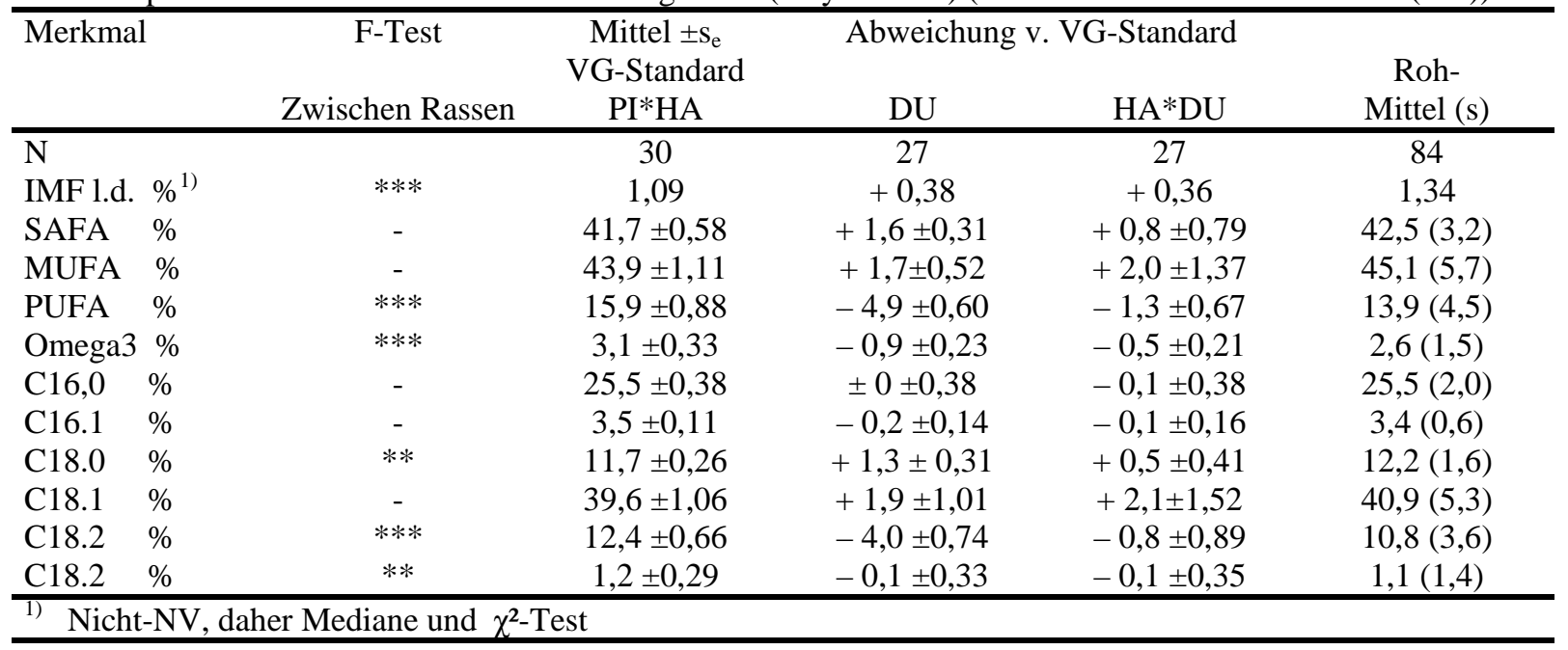

Auch hier zeigten die beiden dänischen Vaterrassen hoch signifikant höhere intramuskuläre Fettgehalte um knapp 0,4 \%, womit sie aber immer noch unter 1,5 \% lagen. Signifikant niedriger als der Vergleischsstandard lagen beide im Anteil PUFA- und Omega3-Fettsäuren zugunsten deutlich höherer SAFA- und MUFA-Anteile. Die we- 
sentlichen Einzelkomponenten dieser Befunde waren C18.0- und C18.2-Fettsäuren. Erstaunlich ist jedoch, dass diese Abweichungen entgegengesetzt zu den im Rückenspeck derselben Schweine gefundenen verlaufen (s. Tab. 5). Eine Erklärung für diesen Befund wäre der höhere intramuskuläre Fettansatz bei DU-Kreuzungen, der vornehmlich durch eine höhere de novo-Synthese von C18.0 und C18.1 Fettsäuren erreicht wird. Die Gegenläufigkeit zwischen Rücken- und intramuskulären Fett könnte mit deren unterschiedlicher Wachstumsgeschwindigkeit zusammenhängen.

\section{Sensorische Bewertung}

Die erzielten Bewertungsnoten und Geschmacksabweichungen sind in Tabelle 7 zusammengestellt.

Tabelle 7

Sensorische Bewertung von Muskel- und Muskel/Speck-Proben (nur 2. Durchgang) (Abweichung vom VGStandard) (Sensoric tests of muscle and muscle/backfat samples (only $2^{\text {nd }}$ trial) (Deviation from standard PI*HA(NN))

\begin{tabular}{|c|c|c|c|c|c|}
\hline \multirow[t]{2}{*}{ Merkmal } & \multirow{2}{*}{$\begin{array}{l}\text { F-Test } \\
\text { Zwischen } \\
\text { Rassen }\end{array}$} & \multirow{2}{*}{$\begin{array}{l}\text { Mittel } \pm \mathrm{s}_{\mathrm{e}} \\
\text { VG-Standard } \\
\text { PI*HA }\end{array}$} & \multicolumn{2}{|c|}{ Abweichung v. VG-Standard } & \multirow{2}{*}{$\begin{array}{c}\text { Roh- } \\
\text { Mittel (s) }\end{array}$} \\
\hline & & & DU & HA*DU & \\
\hline $\mathrm{N}$ & & 22 & 23 & 22 & 67 \\
\hline Saftigkeit ${ }^{1)}$ & $*$ & $3,3 \pm 0,23$ & $-0,6 \pm 0,17$ & $-0,3 \pm 0,17$ & $3,0(0,7)$ \\
\hline Zartheit $^{1)}$ & $* *$ & $3,8 \pm 0,21$ & $0,8 \pm 0,18$ & $-0,2 \pm 0,19$ & $3,5(0,8)$ \\
\hline Aroma m.l.d. ${ }^{1)}$ & - & $2,9 \pm 0,15$ & $-0,1 \pm 0,11$ & $\pm 0 \pm 0,14$ & $2,9(0,4)$ \\
\hline Aroma M/Sp. ${ }^{1)}$ & - & $3,2 \pm 0,19$ & $-0,2 \pm 0,17$ & $\pm 0 \pm 0,20$ & $3,1(0,6)$ \\
\hline Gesamteindruck $^{1)}$ & $\circ$ & $3,1 \pm 0,17$ & $-0,3 \pm 0,13$ & $\pm 0 \pm 0,16$ & $3,0(0,5)$ \\
\hline Schweinearoma ${ }^{2)}$ & - & $2,9 \pm 0,02$ & $+0,1 \pm 0,02$ & $\pm 0 \pm 0,02$ & $2,9(0,1)$ \\
\hline Geschmack sauer²) & - & $2,3 \pm 0,06$ & $+0,1 \pm 0,06$ & $+0,2 \pm 0,06$ & $2,4(0,3)$ \\
\hline Grillverlust \% & $\circ$ & $25,6 \pm 0,83$ & $+1,4 \pm 0,70$ & $+0,9 \pm 0,63$ & $26(2,9)$ \\
\hline Sonst. Bem. ${ }^{3)}$ m.l.d. \% & - & $21,2 \pm 4,67$ & $-8,9 \pm 3,69$ & $-12,1 \pm 3,65$ & $14,4(2,4)$ \\
\hline Fremdart.Eindr. ${ }^{4)}$ m.l.d. \% & - & $13,7 \pm 3,22$ & $-3,5 \pm 2,71$ & $+6,8 \pm 3,79$ & $14,7(2,4)$ \\
\hline M/Sp. ${ }^{4)} \%$ & - & $18,9 \pm 4,56$ & $-6,4 \pm 4,35$ & $+1,6 \pm 4,73$ & $17,2(3,7)$ \\
\hline \multicolumn{6}{|c|}{$\begin{array}{l}\text { 1) sensorische Noten: } 1 \text { = schlechteste ...... } 6 \text { = beste Bewertung } \\
\text { 2) Schweinearoma: } 1 \text { = stark, } 3=\text { normal } \\
\text { 3) Bemerkungen: fade, metallisch, DFD-typisch } \\
\text { 4) fremdartige Eindrücke: süßlich, bitter, sauer, ölig, tranig, fischig, ranzig, leimig oder ekelig }\end{array}$} \\
\hline
\end{tabular}

Signifikante Rassenunterschiede wurden in diesem Test nur bei der Beurteilung von Zartheit, Saftigkeit und mit Abstrichen beim Gesamteindruck gefunden. Bei allen drei Kriterien waren die DU-Nachkommen hoch signifikant schlechter als der PI*HA(NN)Vergleichsstandard, und die HA*DU-Nachkommen lagen dazwischen bzw. gleichauf mit letzterem. Nennenswerte Aromaunterschiede gab es nicht, und der Grillverlust lag bei beiden dänischen Kreuzungen um 1,4 \%zw. 0,9 \% höher als beim deutschen Standard. Fremdartige Eindrücke waren allerdings bei DU-Kreuzungen geringer als bei den beiden anderen genetischen Gruppen, jedoch konnten alle diese Unterschiede an dem kleinen Datenmaterial nicht statistisch abgesichert werden.

\section{Diskussion und Schlussfolgerungen}

Hauptgegenstand dieser Diskussion soll es sein herauszuarbeiten, inwieweit die Befunde der großen niedersächsischen Feldstudie durch diese weitergehenden Einzeltieruntersuchungen ergänzt und möglicherweise auch relativiert werden können. Dabei muss immer im Auge behalten werden, dass in diesem Datensatz mit nur 4 der insgesamt 10 Väter-Nachkommenschaften je Kreuzung durchaus zufällige genetische Unterschiede zum Gesamtmaterial auftreten konnten. Die eigentlichen Rassenunter- 
schiede sollten daher am Gesamtmaterial zuverlässiger geschätzt worden sein, aber die hier erhobenen zusätzlichen Merkmale können wertvolle Interpretationshilfen liefern.

\section{Zuwachsleistungen und Energieaufwand}

$\mathrm{Zu}$ diesem Leistungskomplex konnten im Praxisversuch nur das warme Schlachtgewicht und das Alter bei der Schlachtung erhoben werden, woraus sich die sog. NettoLebenstagszunahme errechnen ließ (PAULUS et al., 2000), während im vorliegendem Exaktversuch individuelle Zunahmen und Futteraufnahmen im Mastabschnitt von 30 $120 \mathrm{~kg}$ Lebendgewicht gemessenen werden konnten (s. Tab. 3). Direkt vergleichbare Wachstumsparameter gab es also nicht, dennoch waren die DU-Nachkommen auch hier beim täglichen Lebend- und Schlachtgewichtszuwachs im Mastabschnitt signifikant überlegen. Gestört wird die Übereinstimmung jedoch durch die PI(PP)-Stichprobe dieses Teildatensatzes, die offenbar zufällig 4 im Zunahmeniveau stark überdurchschnittliche Eber als Väter enthielt. Deshalb können die führenden Netto- und „Fleisch“-Zunahmen dieser Studie nicht verallgemeinert werden, wie auch die hohen Standardfehler für beide PI-Nachkommengruppen nahelegen. Sehr deutlich fällt aber der hoch signifikant größere Knochenanteil aller drei genetischen Gruppen gegenüber der feinknochigen PI(NN) und der Vergleichsgruppe ins Gewicht, er nivelliert die „Fleisch“-Zunahmen aller vier Kreuzungen auf nahezu gleiche Werte. Die mit Abstand beste Energieverwertung zeigten wieder DU-Nachkommen, und auch die besonders homogenen (HA*DU)-Nachkommen waren signifikant besser, während die herausgehoben wüchsige PI(PP)-Stichprobe wegen einer besonders hohen Streuung nicht signifikant vom Standard abwich. Allerdings täuscht dieses eingeschränkte Material bei den dänischen Kreuzungen eine Homogenität in den Mastleistungen vor, die im Gesamtmaterial nicht gefunden wurde, dort zeigten die DU-Kreuzungen in der Nettozunahme mit Abstand die größte Streuung (PAULUS et al., 2000).

Als Fazit lässt sich für die Mastleistung ziehen, dass zwar die Lebendgewichtszunahme durch DU-Väter deutlich erhöht werden kann, wovon aber ihr ebenso deutlich höherer Knochenanteil gegenüber den feinknochigen PI(NN)- und Standard-Nachkommen wenig im „Restzuwachs“ übrig lässt. DU-Eber scheinen dennoch bei der hier praktizierten isoenergetischen Fütterung die Energieverwertung ihrer Nachkommen signifikant zu verbessern.

\section{Schlachtkörperwert}

Vergleicht man den Fleischanteil nach Bonner Formel in dieser Studie mit dem FOMFleischanteil im Gesamtmaterial, so ergaben sich die gleichen Abstände zwischen PI(PP)- mit $+1,4 \%$ und DU-Nachkommen mit $-0,7 \%$, also eine Differenz von über 2 $\%$ zugunsten der ersteren. Stressresistente PI(NN)-Nachkommen folgten sicher an zweiter Stelle im Fleischanteil und rangierten in der Rückenmuskelfläche sogar leicht vor denen der PI(PP)-Eber, während die DU-Nachkommen darin besonders weit unter allen anderen Kreuzungen lagen. Im Flächenverhältnis am Rückenquerschnitt waren die Unterschiede geringer, hier hoben sich nur die PI(PP)-Nachkommen signifikant ab, diese Überlegenheit erreichte im geschätzten Fleischanteil des Bauches sogar +1,8 \%. Im Anteil wertvoller Teilstücke setzte sich diese Tendenz fort, Nachkommen von PI(PP)-Ebern hatten 1 - 1,5 kg (1,5 - 2 \%) mehr davon als die von DU-Ebern, und die von PI(NN)-Ebern lagen etwa in der Mitte zwischen diesen beiden (GLODEK, 1999, PAULUS, 1999). Nicht ganz repräsentativ ist die hier gefundene Streuung im MFA(BF) für die im FOM-Fleischanteil in der Feldstudie, denn dort zeigten die DU- 
Nachkommen einen doppelt so hohen Wert wie PI(NN)-Nachkommen, während dieser im Anteil wertvoller Teilstücke in beiden Studien etwa gleich hoch war.

Das Fazit über den Schlachtkörperwert der hier verglichenen Kreuzungen ist, dass stressanfällige PI-Eber zwar zu Beginn der MHS-Sanierungsphase noch fleischreichere Nachkommen brachten als ihre reinerbigen PI(NN)-Brüder, dass der Unterschied aber nicht wie in diesem Teilmaterial über $1 \%$ beträgt, sondern nur etwa die Hälfte. Dagegen brachte der Einsatz von dänischen DU-Ebern etwa um 2 \% niedrigere Anteile an Muskelfleisch (nach FOM wie BF) und an wertvollen Teilstücken. Inzwischen sind jedoch erhebliche Zuchtfortschritte bei stressresistenten Pietrains gemacht worden, und bei DU-Ebern eröffnet die gefundene Variation zwischen ihnen erhebliche Selektionsspielräume, um deren Nachteile im Fleischanteil abzubauen.

\section{Fleischbeschaffenheit}

Vergleicht man die in dieser Studie gefundenen Rassenunterschiede (s. Tab. 4) in vergleichbaren Qualitätskriterien mit denen der Feldstudie (GLODEK, 1999, LAUBE, 2000), so findet man eine größenordnungsmäßig sehr gute Übereinstimmung. Es zeigt sich in den frühpostmortalen PH-Werten, im LF24h wie in den Gehalten an Wasser, Fett, Protein und Glykogen, dass die PI(PP)-Nachkommen die typischen PSE- und die $\mathrm{HA}$ enthaltenden die typischen $\mathrm{RN}^{-}$-Veränderungen hoch signifikant ausbilden, während alle MHS(NN)-Genotypen ziemlich einheitlich gute Fleischqualität zeigten und alle nicht HA enthaltenden vergleichbar gute Glykogen-, Wasser- und Proteingehalte im Fleisch. Selbst im intramuskulären Fettgehalt zeigten sich nahezu gleiche Differenzen, die für DU- und etwas geringer auch für HA*DU-Eber signifikant überlegen und mindestens für PI(PP)-Eber gesichert unterlegen waren. Während in der Feldstudie bei DU-Nachkommen noch der Mittelwert von etwa $2 \%$ erreicht wurde, lag er in diesem Teilmaterial sogar unter 1,5\%.

Die Farb- und Helligkeitsmessungen haben weder mit dem OPTO-Verfahren in dieser wie mit der Minoltamessung in der Feldstudie deutlichere Qualitätsunterschiede identifiziert als die bisher betrachteten Hilfsmerkmale. Ein verarbeitungstechnologisch wichtiges Kriterium ist der in dieser Studie zusätzlich erhobene Drip-Verlust, der am 2.-4. Tag p.m. gemessen die deutlichsten Werte von $+1,3 \%-1,6 \%$ Gewichtsverlust für mischerbig stressanfällige PI(PP)-Nachkommen auswies aber auch die DU-Nachkommen mit $-0,6 \%$ aus den übrigen stressstabilen Kreuzungen (zwischen $+0,3 \%$ bis $+0,5 \%$ liegend) noch klar heraushob.

Das Fazit für die Fleischbeschaffenheit der verglichenen Kreuzungen lautet, dass nur reinerbig stressresistente Endprodukte weitgehende PSE-Freiheit garantieren und dass spätpostmortale Entgleisungen nur mit Produkten ohne das $\mathrm{RN}^{-}$-Gen vermieden werden können. Bezüglich des intramuskulären Fettgehaltes, der hier bei allen Kreuzungen bereits deutlich unter den von Experten geforderten 2 - 2,5 \% lag, soll ein Urteil erst gefällt werden, nachdem die Ergebnisse des sensorischen Tests besprochen sind (s. 4.6).

\section{Fettsäurenmuster im Speck und Muskel}

Der Diskussion des Fettsäurenmusters müssen zwei wesentliche Fakten vorausgeschickt werden:

1) Die Fettsäurenzusammensetzung des Fettes und des Fleisches hängt in starkem Maße von der Futterzusammensetzung ab. KRATZ (2003) fand Korrelationen von 0.83 bis 0.95 zwischen Fettsäureaufnahme und deren Gehalt im Rückenspeck und im Rückenmuskel. Die Unterschiede zwischen den Fütterungsgruppen mit ver- 
schiedenen Fettsäurezulagen waren daher etwa zehnmal so groß wie die zwischen den Vaterrassen. Herausragende Steigerungen des C18.3- und Omega3-Gehalts wurden durch Beigabe von Leinöl erzielt, während PUFA-Anteile auch durch Sojaöl (neben Leinöl) und MUFA durch Olivenöl besonders gesteigert werden konnten.

2) Die relativ größten Rassenunterschiede ergaben sich bei PUFA- und Omega3Fettsäuren und deren Hauptkomponenten C18.3- und C18.2-Säuren, aber auch bei diesen waren die Futtereffekte um den Faktor 10 höher als die Rasseneffekte.

Wenn es also darum geht, den Gehalt von Muskelfleisch oder Speck an bestimmten diätetisch interessanten Fettsäuren (z.B. PUFA oder Omega 3) zu steigern, ist dies über die Verabreichung mit dem Futter weitaus wirksamer und schneller zu erreichen als durch Züchtung und Rassenwahl. Die größten Rasseneffekte auf das Fettsäurenmuster im Muskel wurden für DU-Väter gefunden, aber sie vererbten hoch signifikant höhere SAFA- und MUFA-Anteile und entsprechend niedrigere PUFA- und Omega3Anteile an ihre Nachkommen. Alle anderen hier verwendeten Vaterrassen lagen innerhalb des Konfidenzbereichs der (PI*HA)-Standardgruppe, darüber hinaus waren die Einflüsse im Rückenspeck deutlich geringer als im Muskel.

Allgemein geht man davon aus, dass mit steigendem Anteil ungesättigter Fettsäuren im Futter die Oxidationsstabilität im Rancimattest, gemessen als Induktionszeit (bis zum Entstehen erster flüchtiger Oxidationsprodukte mit qualitätsmindernden Eigenschaften), sinkt. Diese Erwartung bestätigte sich auch beim Vergleich der verschiedenen Futtergruppen, welche an anderer Stelle publiziert werden. Die Rassenunterschiede in beiden Versuchsdurchgängen waren aber sehr klein und statistisch nicht abzusichern, so dass auf eine weitere Diskussion hier verzichtet wird.

\section{Sensorische Bewertung}

Dieser sensorische Test dürfte einerseits durch das niedrige Niveau im intramuskulären Fettgehalt ( $<1,5 \%$ ) und zum anderen durch die weitgehend fehlenden Unterschiede im Fettsäurenmuster und im Rancimattest zwischen den beteiligten Rassen zu erklären sein. Dass dennoch signifikante Rassenunterschiede in den wichtigsten Qualitätsmerkmalen Zartheit und Saftigkeit gefunden wurden, die im Gesamteindruck und im Grill-Verlust ebenfalls die Sicherung fast erreichten, spricht für die Genauigkeit der subjektiven Merkmalserhebung an der BAFF in Kulmbach. Erstaunlich ist allerdings, dass nicht die beiden dänischen Kreuzungen mit signifikant höheren intramuskulären Fettgehalten die bessere Bewertung erfuhren, sondern das (PI*HA(NN))-Standardprodukt als zarter und saftiger eingestuft wurde. Da leider PI-Nachkommen in dieser Studie nicht getestet wurden, konnte weder deren Rasseneinfluss noch der des MHS-Status der PI-Väter untersucht werden. Der Vergleich der (HA*DU)- mit den DU-Nachkommen legt aber nahe, dass vielleicht der HA-Anteil einen positiven Effekt auf diese Qualitätsparameter ausübte. Dieser Frage sollte in weiteren Untersuchungen mit verschiedenen PI-, HA- und DU-Kreuzungen intensiv nachgegangen werden.

\section{Schlussfolgerungen für die deutsche Schweinzucht}

Bei vorsichtiger Interpretation dieser lediglich auf Nachkommen von nur vier Vätern je Rasse beruhenden Daten kann man sagen, dass sie die Ergebnisse der Praxisstudie an wesentlich größerem Datenmaterial bestätigen und mit anspruchsvolleren Qualitätskriterien untermauern:

1) Nachkommen stressanfälliger Pietraineber haben zwar höhere Fleischanteile im Schlachtkörper aber um so viel schlechtere Fleischbeschaffenheitskriterien, vor al- 
lem im PSE-Bereich, dass sie selbst für Standardmärkte bei heutigen Qualitätsanforderungen nicht mehr in Frage kommen.

2) Dagegen erfüllen die Nachkommen reinerbig stressresistenter Pietraineber diese Qualitätsanforderungen voll, jedoch muss vor allem die Zuwachsleistung ihrer Produkte genetisch verbessert werden.

3) Dänische Duroceber vererben zwar deutlich bessere Masteigenschaften, allerdings auf Kosten des Fleischanteils und damit des Markterlöses. Wollte man sie zur Erzeugung von Edelmarkenprodukten einsetzen, so reicht ihr intramuskulärer Fettanteil jedoch nicht mehr aus, um in die sensorisch fühlbaren Bereiche von über $2 \% \mathrm{zu}$ gelangen.

4) Zur Herstellung von Edelprodukten mit definierten Anteilen besonders erwünschter ungesättigter und Omega 3-Fettsäuren sind die genetischen Rassendifferenzen zu klein, sie lassen sich wesentlich schneller und genauer mit der richtigen Fettergänzung im Mastfutter erreichen.

5) Vaterrassen mit einem Hamsphireanteil führen wegen des dominanten $\mathrm{RN}^{-}$-Gens bei ihren Nachkommen zu spätpostmortalen Qualitätsmängeln, die besonders durch Saftverluste bei der Kochschinkenherstellung auffallen.

Weitere Forschungsarbeiten sind vor allem auf dem Gebiet der besonderen Qualitätseigenschaften nach der MHS-Sanierung und bei der Erhöhung des Proteinansatzes ohne negative Auswirkungen auf die Fleischqualität erforderlich.

GLODEK, P.:

\section{Literatur}

Die Eignung von Schweinekreuzungen mit besonderen Qualitätseigenschaften zur Verbesserung des deutschen Markenschweineangebots. Abschlussbericht Institut für Tierzucht und Haustiergenetik, pp. 62. 1999

KRATZ, R.:

Einfluss verschiedener Fettquellen in der Ernährung von Schweinen unterschiedlicher Genetik auf den Protein- und Lipidansatz, das Fettsäurenmuster verschiedener Teilstücke und die Fleischbeschaffenheit. Diss. FB09, Gießen, 2003

LAUBE, S.:

Die Eignung spezieller Schweinekreuzungen zur Qualitätsverbesserung von Markenschweinefleisch unter besonderer Berücksichtigung von MHS-Status, Hampshirefaktor und intramuskulärem Fettgehalt. Diss. TiHo Hannover, 2000

LAUBE, S.; HENNING, M.; BRANDT, H.; KALLWEIT, E.; GLODEK, P.:

Die Fleischbeschaffenheit von Schweinekreuzungen mit besonderen Qualitätseigenschaften im Vergleich zum heutigen Standard- und Markenschweineangebot. Arch. Tierz., Dummerstorf 43 (2000), 463-476

PAULUS, E.-D.:

Die Fleischleistung und der Markterlös von Schweinekreuzungen mit besonderen Qualitätseigenschaften im Vergleich zum heutigen Standard- und Markenschweineangebot. Diss. Ldw. Fak., Göttingen. Cuvillier Verlag Göttingen, ISBN 3-89712-717-2, 2000

PAULUS, E.-D.; HENNING, M.; BRANDT, H.; KALLWEIT, E.; GLODEK, P.:

Die Fleischleistung und der Markterlös von Schweinekreuzungen mit besonderen Qualitätseigenschaften im Vergleich zum heutigen Standard- und Markenschweineangebot. Züchtungskunde 72 (2000), 290-307

Eingegangen: 28.10.2003

Anschriften der Verfasser

Prof. em. Dr. PETER GLODEK

Institut für Tierzucht und Haustiergenetik

Albrecht-Thaer-Weg 3

D-37075 Göttingen
Akzeptiert: 10.12.2003

Prof. Dr. GERHARD FLACHOWSKY, Dr. EDGAR SCHULZ, Dr. RÜDIGER KRATZ Institut für Tierernährung der FAL Bundesallee 50 D-38116 Braunschweig 